\title{
Tuning the Size of Gold Nanoparticles with Repetitive Oxidation-reduction Cycles
}

\author{
Steve Y. Rhieu, VytasReipa* \\ Biosystems and Biomaterials Division, National Institute of Standards and Technology, Gaithersburg, MD 20899, USA \\ *Corresponding author: steve_rhieu@alumni.brown.edu;vytas@nist.gov
}

Received February 12, 2015; Revised March 14, 2015; Accepted March 31, 2015

\begin{abstract}
A simple method to control the size of gold nanoparticles (AuNP) using repetitive oxidation-reduction cycles is described. First, AuNP are shown to be readily immobilized onto an indium-doped tin oxide coated glass surface using cyclic voltammetry nanoparticle containing citrate buffer. Subsequently, the attached AuNPsize can be reduced to a desired level by potential cyclingin the range from $0 \mathrm{~V}$ to $+1.1 \mathrm{~V}$ (vs. Ag/AgCl).Gradual AuNPdiameter decrease was attributed to the formation of gold oxide upon anodic potential sweep and the partial solubilization of the $\mathrm{Au}(\mathrm{III})$ species during subsequent reduction of gold oxide in the absence of gold chelator (e.g., $\mathrm{Cl}^{-}, \mathrm{Br}^{-}$, or $\mathrm{CN}^{-}$) normally necessary for anodic gold dissolution.
\end{abstract}

Keywords: gold nanoparticles, size control, oxidation-reduction, electrochemistry

Cite This Article: Steve Y. Rhieu, and VytasReipa, "Tuning the Size of Gold Nanoparticles with Repetitive Oxidation-reduction Cycles.” American Journal of Nanomaterials, vol. 3, no. 1 (2015): 15-21. doi: 10.12691/ajn3-1-2.

\section{Introduction}

Gold nanoparticles (AuNP) have been widely employed as a functional building block used to modify the surface of an electrode for electro analytical applications. [1,2] Such electrodes demonstrate improved signal-to-noise ratios or enhanced electron-transfer rates between the active site of redox proteins and electrode surfaces, making it an attractive platform to develop potential biosensors and bioreactors. [3,4,5] It also offers a tool to control the electrode properties by adjusting size, shape, and protective monolayers of nanoparticles. [6] Several methods have been developed to bind AuNP to the electrode surfaces using the direct electrocrystallization from the complex gold salts (chlorauric acid $\left(\mathrm{HAuCl}_{4}\right)$ [7,8] and potassium gold cyanide (KAu(CN) $)_{2}$ [9]). Alternatively, connecting soluble AuNP through siloxanebased linkers affords the direct attachment of colloidal AuNP to the electrode surfaces (e.g., indium-doped tin oxide (ITO) coated glass) by the use of covalent interactions. [10,11] However, the bridging organic reagents between the electrode surface and a nanoparticle would hamper the electron-exchange process between them due to the extra charge transfer resistance [2] when the standard electroanalytical methods are used to interrogate electrochemical response of surfaceimmobilized nanoparticles [10,11].

Similar immobilization method was applied to directly measure faradaic responses at a single AuNP using platinum nanoelectrodes. [12] Linker usage was avoided when AuNP are physisorbed using a drop-casting to screen printed [13] or glassy carbon electrodes, [14] that enabled to register particle size-dependent charge-transfer responses. A direct charge exchange between the freely diffusing nanoparticles and the ultramicroelectrode was also demonstrated recently, $[14,16,17,18,19,20]$ despite low diffusion coefficients of nanoparticles in solution. Additionally, a single AuNP electrochemical response was recorded when immobilized on a Pt microelectrode just by repetitive potential scanning in a AuNP suspension. [21] In the present work, we demonstrate a facile method to immobilize AuNP on the ITO coated glass electrodes without resorting to linker molecules or gold salts, and surface attached nanoparticle size control using recurring gold oxidation-reduction cycles in the citrate-containing electrolytes.

\section{Materials and Methods}

ITO coated (Rs $=70-100 \Omega$ ) glass slides were obtained from Delta Technologies, Ltd. (Stillwater, MN). Gold nanoparticles with nominal diameters of $4 \mathrm{~nm}$ and $20 \mathrm{~nm}$ were purchased from Nanopartz, Inc. (Loveland, CO) and British Biocell International (Cardiff, UK), respectively, and characterized by TEM and DLS to have mean diameters of $2.9 \pm 0.5 \mathrm{~nm}$ and $24.3 \pm 2.4 \mathrm{~nm}$, respectively (Figure S4, Supporting Information). All chemicals were of analytical reagent grade (Sigma-Aldrich, St. Louis, MO) and used as received. High-purity water with a resistivity of $18.2 \mathrm{M} \Omega \cdot \mathrm{cm}$ was used in all experiments. Measurements of the gold nanoparticle hydrodynamic radius were performed using a Nicomp Model 380 ZLS DLS system (Particle Sizing Systems Inc., Santa Barbara, CA, USA). Samples were measured in $6 \times 50 \mathrm{~mm}$ glass Kimble Chase disposable culture tubes. Prior to sample 
loading, the tubes were blown with $\mathrm{N}_{2}$ gas and filled with $0.3 \mathrm{ml}$ of the sample. The autocorrelation function was acquired for $3 \mathrm{~min}$ in triplicate and fitted to intensity weighted NICOMP size distribution analysis. Electrochemical measurements were performed using an EG\&G M263A potentiostat/galvanostat (Princeton Applied Research) at room temperature. The electrochemical cell consists of the ITO disk (with an exposed geometric area of $c a .0 .126 \mathrm{~cm}^{2}$ ) working electrode, a platinum wire counter electrode, and a Ag/AgCl (0.197 V vs. NHE) reference electrode. Prior to electrochemical experiments, ITO substrates were cleaned by sonication in ethanol for 15 min, rinsed in acetone, and blown dry with $\mathrm{N}_{2}$. For anodic stripping of the nanoparticles on ITO, the working solution (i.e., $0.1 \mathrm{M}$ hydrochloric acid in $13.3 \mathrm{mM}$ citrate buffer) was purged with $\mathrm{N}_{2}$ for $30 \mathrm{~min}$ and a blanket of $\mathrm{N}_{2}$ was kept over the solution during measurements. All potentials are referred to the Ag/AgCl reference electrode unless stated otherwise.

\section{Results and Discussion}

First, we have recorded cyclic voltammograms (CV) for a bare ITO electrode immersed in $13.3 \mathrm{mmol} / \mathrm{L}$ citrate buffer ( $\mathrm{pH}$ 6.0) solution containing AuNP with $4 \mathrm{~nm}$ nominal diameter. The stability of AuNP in working solution was confirmed prior to running experiments (Figure S1, Supporting Information). Figure 1 compares the CV of the bare ITO electrode priorto (dotted line) and following the introduction of the AuNP at $2.71 \times 10^{13}$ parts per milliliter to the working solution. The voltammetric response shows an anodic current peak at around $+0.85 \mathrm{~V}$ and a cathodic counterpart at around $+0.54 \mathrm{~V}$, which reflect the oxidation of AuNP and the subsequent reduction of the gold oxide species back to metallic gold, respectively. The peak potentials $\left(E_{p}\right)$ for both anodic and cathodic peaks displays a linear dependence with the natural logarithm of scan rate (see inset of Figure 1), demonstrating the charge exchange with the surface immobilized redox entities. Similar scan rate dependencies were observed in previous studies with AuNP [21] or silver nanoparticles [22,23] immobilized on electrode surfaces. We infer that AuNP are being attached on the ITO electrodes used in this study either during the successive scans or potentially via physisorptionas part of seed-mediated growth. [15,24,25] To test the surface attachment, the surface of an electrode was washed with copious amount of deionized water, followed by the cyclic voltammetry scan in the nanoparticle-free citrate buffer solution. As shown in the inset of Figure 2, the CV profile, comparable to the one before washing was recorded. However, the anodic peak at around $+0.85 \mathrm{~V}$ has shifted to a more positive potential. The presence of the surface attached AuNP was also confirmed with scanning electron microscopy (SEM) images and UV-Vis absorbance (Figure S1 and Figure S2, Supporting Information). Repeated potential scans in citrate-containing electrolytes result in both anodic and cathodic current peak decline and their complete disappearance after about 50 scans (see dotted curve in the inset of Figure 2), indicating a gradual loss of the total active gold surface area. Such outcome could be caused by AuNP dissolution (size reduction) during the potential cycling [14,26] or immobilized particle number decrease due desorption from the ITO surface. To verify a second possibility, we have subjected the immobilized AuNPto cyclic voltammetry treatment in the extended potential range from $-0.5 \mathrm{~V}$ to $+1.1 \mathrm{~V}$, thus considerably varying the electrostatic interaction between the negatively charged (capped with citrate or carboxylic acid moieties) AuNP and the ITO electrode. First, the potential cycling was performed from $+0.6 \mathrm{~V}$ to $-0.5 \mathrm{~V}$ at $60 \mathrm{mV} / \mathrm{s}$, i.e. outside the range of $\mathrm{Au}$ oxidation (red trace in Figure 3). Next, 10 roundtrip potential cycles were performed from $+0.7 \mathrm{~V}$ to $+1.1 \mathrm{~V}$ (blue trace on Figure 2), thus excluding the potential range of the previously recorded gold oxide reduction peak (centered at $E=0.54 \mathrm{~V}$, see insert of Figure 2). Following this treatment, we have repeated the scan in the range from $0 \mathrm{~V}$ to $+1.1 \mathrm{~V}$ (black trace in Figure 2). Notably, the recovered voltammogram was almost identical to the one, recorded prior to the wide range potential cycling. Such result clearly invalidates our second hypothesis, and shows that the electrostatic desorption of AuNP is not responsible for the decrease in the current peak charge repeated cycling as shown in the insert of Figure 2.

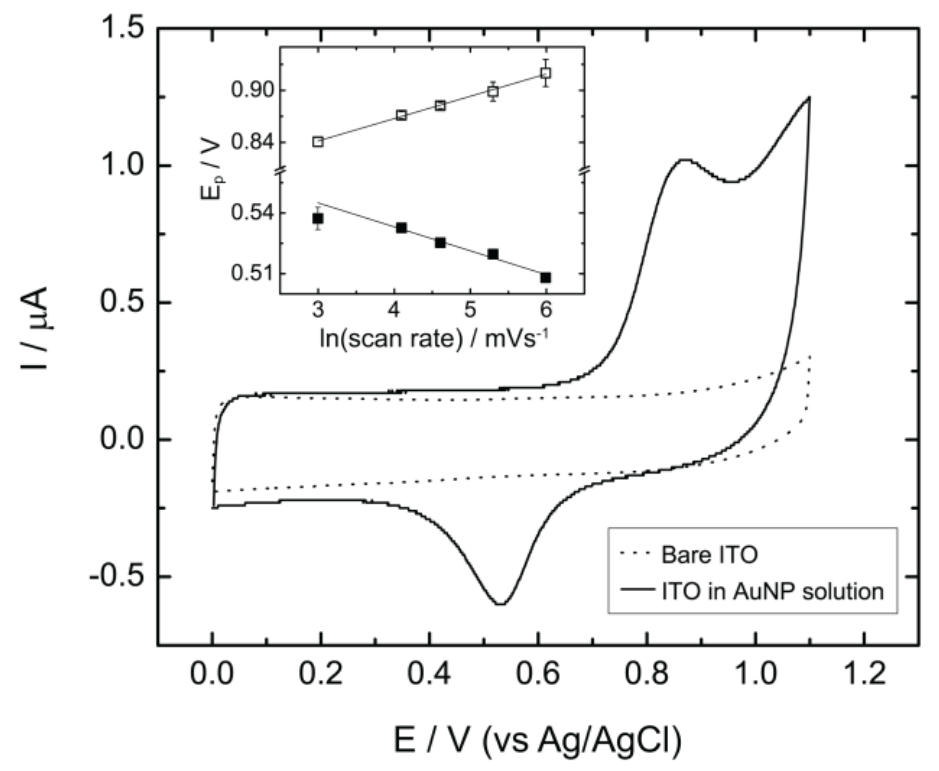

Figure 1. Voltammetric responses of a bare ITO electrode in a $13.3 \mathrm{mmol} / \mathrm{l}$ citrate buffer solution containing either $4 \mathrm{~nm}$ AuNP (solid) or no particles (dotted). Scan rate: $60 \mathrm{mV} \mathrm{s}^{-1}$. Inset: Peak potentials $\left(E_{\mathrm{p}}\right)$ for both anodic and cathodic peak versus the natural logarithm of scan rate 


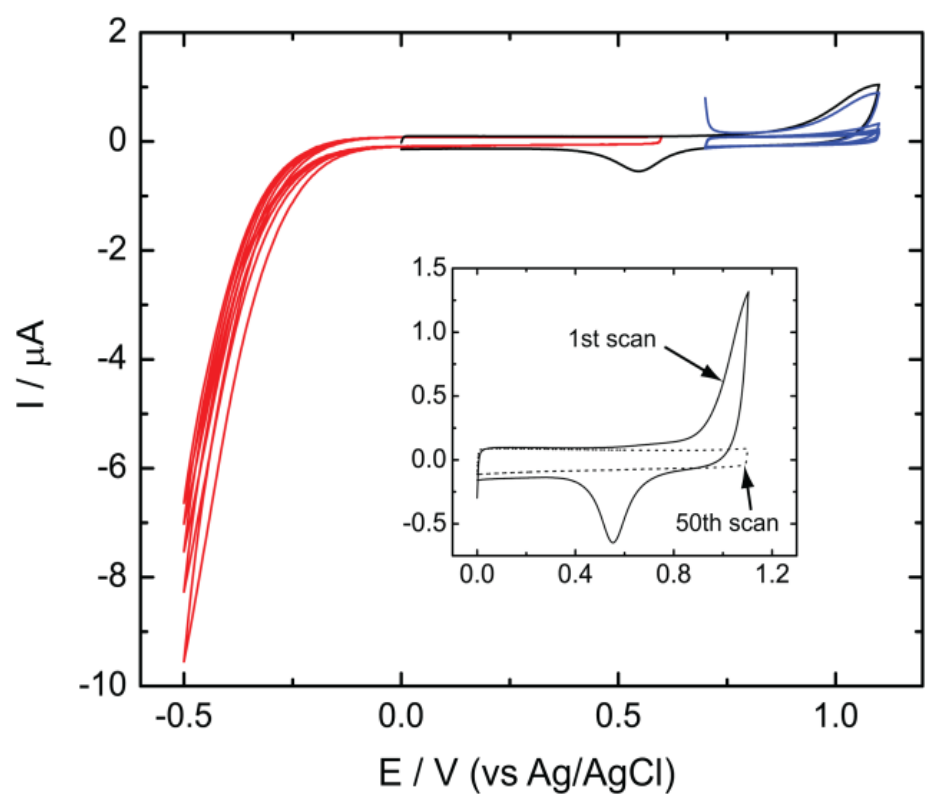

Figure 2. Voltammetric responses of aAuNP-modified ITO electrode at different potential windows: $-0.5 \mathrm{~V}$ to $+0.6 \mathrm{~V}$ (red line), $0 \mathrm{~V}$ to $+1.1 \mathrm{~V}$ (black line), $+0.7 \mathrm{~V}$ to $+1.1 \mathrm{~V}$ (blue line). Scan rate: $60 \mathrm{mV} \mathrm{s}$. . Inset: voltammetric responses aAuNP-modified ITO electrode in a $13.3 \mathrm{mM}$ citrate buffer solution: $1^{\text {st }}$ scan (solid line) and $50^{\text {th }}$ scan (dotted line)
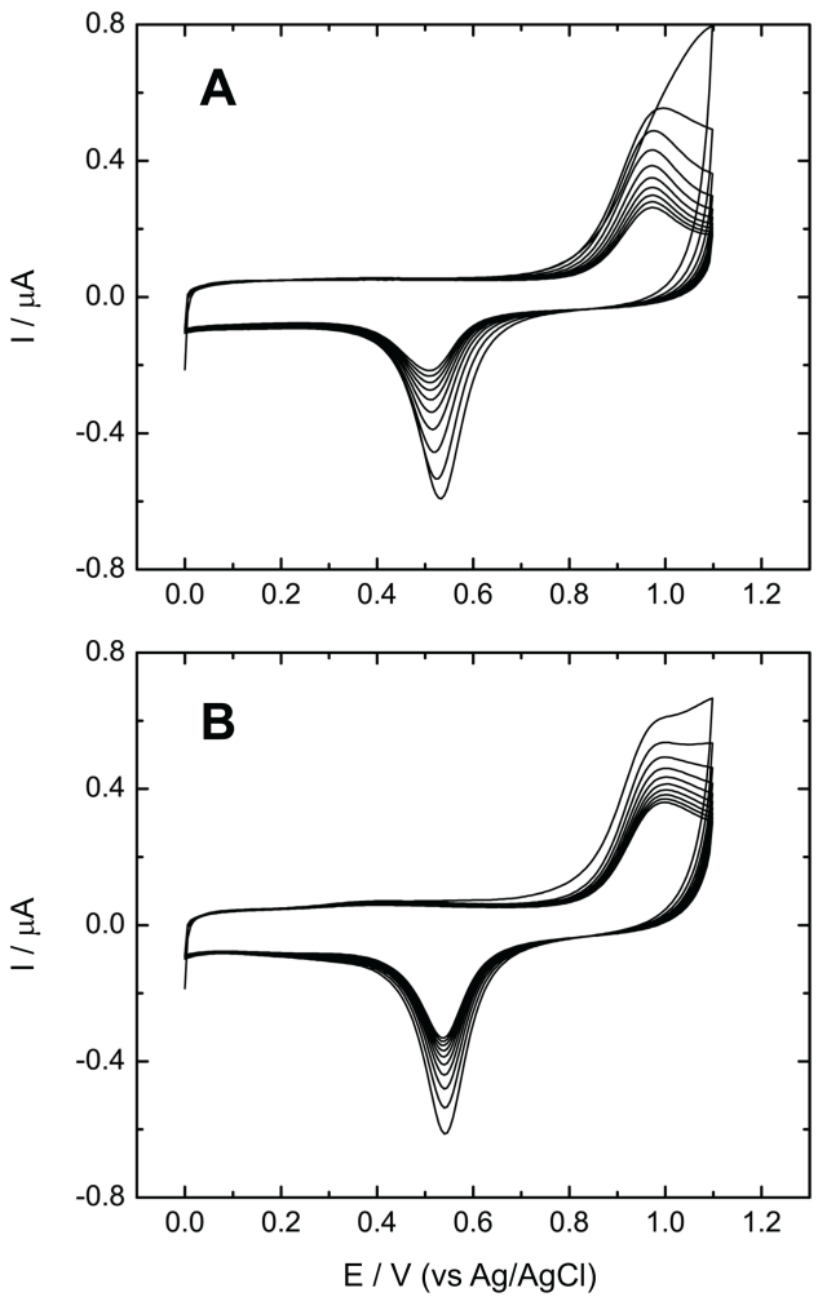

Figure 3. Successive cyclic voltamograms (first 10 cycles) obtained with (A) $4 \mathrm{~nm}$ and (b) $20 \mathrm{~nm}$ AuNP-modified ITO electrodes in $13.3 \mathrm{mM}$ citrate buffer solution. Scan rate: $60 \mathrm{mV} \mathrm{s}^{-1}$

It indicates that the gradual charge loss during potential cycling is consistent with the ITO surface attached AuNP dissolution. On the other hand, a gold chelator (e.g. $\mathrm{Cl}^{-}$, $\mathrm{Br}^{-}$, or $\mathrm{CN}^{-}$) normally is needed for anodic gold

dissolution reaction to progress. Working solution used in this study, however, did not contain such ligands and, moreover, the observed CV profiles were typical for gold oxide formation and reduction of gold oxide during the reverse scans (Figure 2). Gold oxide reduction in weak non-complexingacidic solutions proceeds according to: [27]

$$
\mathrm{Au}(\mathrm{OH})_{3}+3 \mathrm{H}^{+}+3 \mathrm{e}^{-} \rightarrow \mathrm{Au}+3 \mathrm{H}_{2} \mathrm{O}
$$

The gold oxide reduction reaction in Eq.(1) is perceived to be a solid-solid reaction and does not contain soluble intermediates. However, several previous studies found evidence that soluble $\mathrm{Au}(\mathrm{III})$ species can actually form upon gold oxide reduction in electrolytes with no gold complexinganions. Cadle et al. have detected up to 3\% of soluble gold species after a single oxidation-reduction cycle of the gold disk in sulfuric acid solutions using a rotating Pt ring. [26] A later quartz microbalance study by Shackleford et al. reported gold electrode mass loss and surface roughening while following a series of slow potential cycles in nitric acid based electrolytes. [28]

To further explore particle size decrease upon consecutive oxidation-reduction in citrate-containing electrolytes, we compared the coulometric data from AuNP(nominal diameters of $4 \mathrm{~nm}$ and $20 \mathrm{~nm}$ ) attached on the ITO surface. First, AuNP were immobilized on the ITO surface by successive scans from $0 \mathrm{~V}$ to $+1.1 \mathrm{~V}$ using colloidal solutions at concentrations $C_{4 \mathrm{~nm}}=2.71 \times 10^{13}$ part $/ \mathrm{mL}$ and $C_{20 \mathrm{~nm}}=1.26 \times 10^{12}$ parts per milliliter. Following washing with deionized water, the electrodes were immersed in citrate buffer solutions for further analysis. Figure 3A shows CV of ten consecutive scans of ITO/AuNP with a nominal diameter of $4 \mathrm{~nm}$, showing steady decay in both anodic and cathodic peak amplitudes. The active gold area gets smaller with each potential cycle, indicating that gold oxide monolayer is formed during potential scan to $+1.1 \mathrm{~V}$ and a small fraction of gold is solubilized when gold oxide is reduced (current peak at $\mathrm{E}_{\mathrm{p}}$ $=+0.54 \mathrm{~V}$, Figure 3). The same holds true for AuNP with a nominal diameter of $20 \mathrm{~nm}$, although the rate of charge 
decay with respect to the scan number was considerably slower than for $4 \mathrm{~nm}$ AuNP (Figure 3B). Also, the cathodic and anodic peak potentials measured for both 4 $\mathrm{nm}$ and $20 \mathrm{~nm}$ AuNP were shifted relative to the bulk gold oxidation (Figure S4, Supporting Information).Such sizedependent AuNP oxidation potential shifts were observed in previous studies $[11,12]$ using surface attached particles and suggested a theoretical model based on free energy changes with the metal surface area.

The immobilized AuNP particle size could be directly determined from the coulometry data, following the approach as described. [29] First, the total Au particle surface area was estimated by integrating the charge under the gold oxide reduction peak following the first potential cycle in the citrate-containing electrolytes (black traces in Figure 4), using a value of $386 \mu \mathrm{C} / \mathrm{cm}^{2}$ required to reduce the monolayer of gold oxide. [30]Next, a total gold atom number of the surface-attached AuNP's was determined by stripping voltammetry ina $0.1 \mathrm{~mol} / \mathrm{LHCl}$ solution. Here, several potential cycles between $+0.7 \mathrm{~V}$ and $+1.3 \mathrm{~V}$ (vs $\mathrm{Ag} / \mathrm{AgCl}$ ) were applied (red traces in Figure 4) until complete gold dissolution and the required charge was integrated. As evident from the current traces in Figure 4, immobilized AuNP's are completely solubilized following the first two potential cycles.
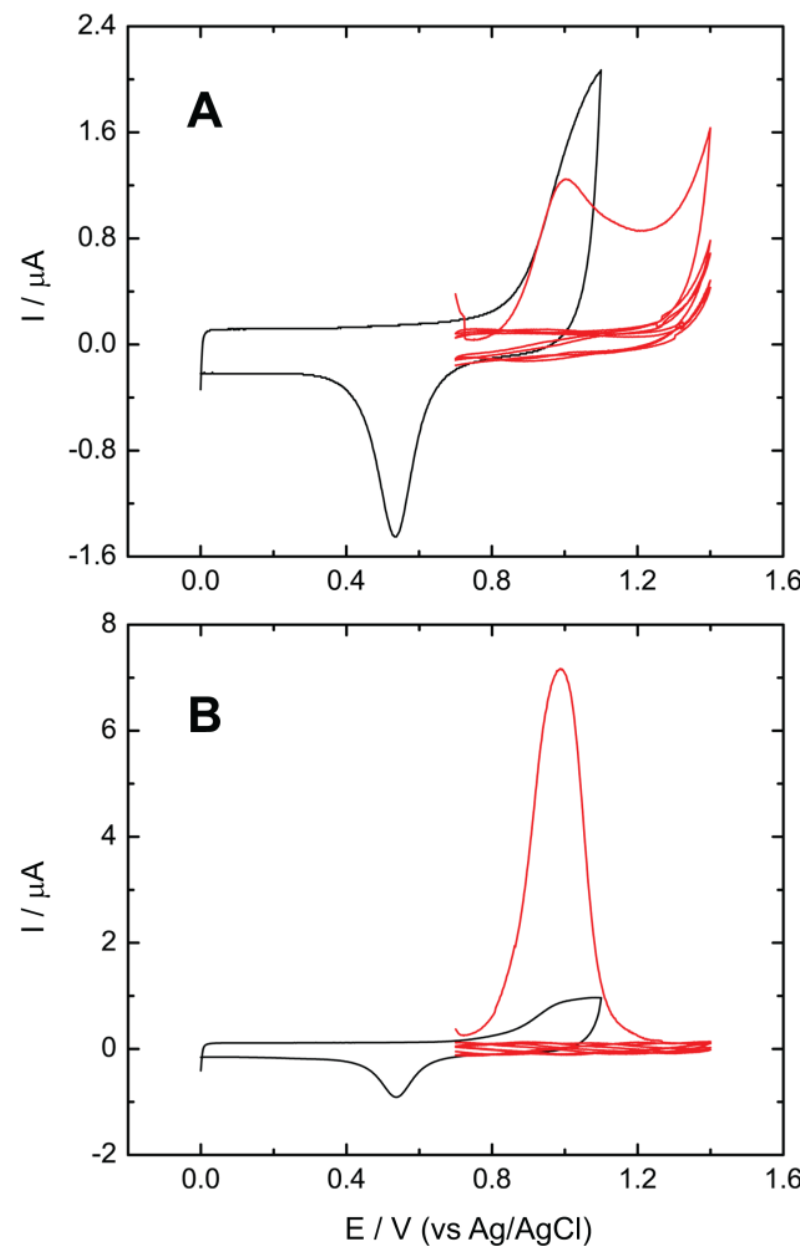

Figure 4. Voltammetric responses of (A) $4 \mathrm{~nm}$ and (B) $20 \mathrm{~nm}$ AuNPmodified ITO electrodes before (black) and after (red) carrying out anodic stripping of the nanoparticles in $0.1 \mathrm{M} \mathrm{HCl}$. All the solutions were deoxygenated by purging with $\mathrm{N}_{2}$ for at least $30 \mathrm{~min}$ and the experiments were carried out under $\mathrm{N}_{2}$ atmosphere. Scan rate: $60 \mathrm{mV} \mathrm{s}^{-1}$

The anodic stripping charge was then used to estimate the amount of total gold immobilized on ITO using $1.9 \pm$
0.1 electrons transferred per gold atom. [14] Taken together with the earlier determined total particle surface area, this allows us tomeasure the radius of the immobilized particle and their surface concentration. The summary of measurement results is provided in Table 1. The obtained particle diameter values are in good agreement with particle diameters from transmission electron microscopy (TEM) images and dynamic light scattering (DLS)measurements (Figure S4, Supporting Information).

Table 1. Size and concentration of AuNP attached on ITO electrodes measured by coulometry ${ }^{\mathrm{a}}$

\begin{tabular}{cccc}
\hline $\begin{array}{c}\text { AuNP } \\
\text { nominal } \\
\text { diameter } \\
(\mathrm{nm})\end{array}$ & $\begin{array}{c}\text { Particle surface } \\
\text { concentration (parts } \\
\text { per square } \\
\text { centimeter) }\end{array}$ & $\begin{array}{c}\text { Average } \\
\text { particle } \\
\text { diameter }(\mathrm{nm})\end{array}$ & $\begin{array}{c}\text { Radius } \\
\text { differential } \Delta r \\
(\mathrm{~nm})\end{array}$ \\
\hline 4 & $(2.82 \pm 0.15) \times 10^{11}$ & $3.53 \pm 0.27$ & $0.2 \pm 0.04$ \\
20 & $(4.79 \pm 0.38) \times 10^{9}$ & $20.74 \pm 2.31$ & $0.74 \pm 0.14$ \\
\hline
\end{tabular}

${ }^{\mathrm{a}}$ Uncertainties are calculated as the estimated standard deviation from the mean from three independent measurements.

Gold oxidation depth is determined by the anodic potential scan limit, [31] therefore a particle of radius $r$ would lose the $\Delta r$ after each oxidation-reduction cycle (cycle number $n$ ), thus the spherical particle surface area $S$ can be expressed as:

$$
S=4 \pi(r-n \Delta r)^{2}
$$

The total gold oxide reduction charge $(C)$ will change after each cycle according to:

$$
C=4 \pi N c\left(r^{2}-2 r \Delta r+n^{2} \Delta r^{2}\right)
$$

where $N$ is the number of particles and $c$ is the gold oxide monolayer reduction charge $\left(386 \mu \mathrm{C} / \mathrm{cm}^{2}\right)$. [30] A nonlinear regression fit of the experimentally determined gold oxide reductioncharge (Q) vs. the number of potential cycles $(n)$ to this model is presented in Figure 5.

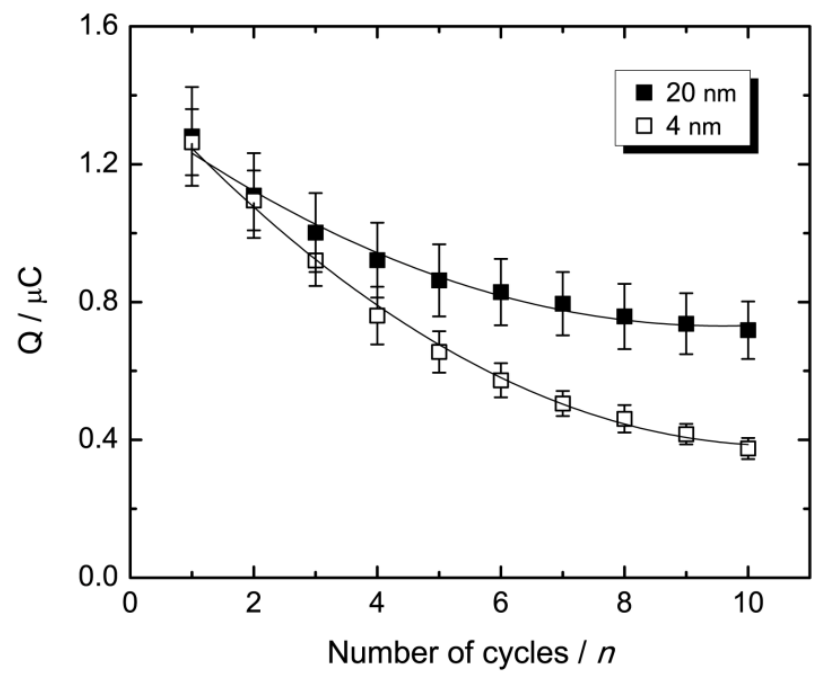

Figure 5. Gold oxide reduction charge (Q) as a function of cycle number $(n)$. Error bars represent on standard deviation from three independent experiments

Experimental data fit to this model allows us to estimate a value of radius differential $\Delta r$ for cyclic voltammetry experiments with $4 \mathrm{~nm}$ and $20 \mathrm{~nm}$ AuNP (Table 1). The $\Delta r$ values for AuNP mirror the total mass loss with each cycle, which is comparable with the earlier data of Cadle et al. who reported a 3\% material loss when 
using a rotating ring-disk gold electrode in sulfuric acid. [26] It is noteworthy that smaller particles are more efficiently attached to the ITO surface asillustrated by more than twofold increase in particle surface concentration $\operatorname{ratio}\left(N_{(4 \mathrm{~nm}} \quad\right.$ AuNP $) / N_{(20 \mathrm{~nm}} \quad$ AuNP $\left.)=58.9 \pm 4\right)$ compared to their respective concentrations in the electrolytes $\left(C_{(4 \mathrm{~nm} \mathrm{AuNP})} / C_{(20 \mathrm{~nm} \mathrm{AuNP})}=21.5 \pm 3\right)$.

\section{Conclusions}

In summary, we have described a simple method to directly attach citrate-terminated gold nanoparticle to the ITO surface using potential cycling in the gold oxidation range. The average size of the surface immobilized particles measured by $\mathrm{CV}$ and stripping voltammetry mirrorsparticle size information, obtained from the electron microscopy and manufacturers data. A repetitive potential cycling in the citrate-containing electrolytes offers a simple and accurate methode to shrink the surface attached gold nanoparticle size to the desired value.

\section{Acknowledgements}

This research was conducted while S.Y.R. held a National Research Council Research Associate ship at the National Institute of Standards and Technology. We thank Drs. Kanokwan Nontapot and Charles J. Choi of NIST Center for Nanoscience and Technology for their assistance with the transmission electron microscopy and scanning electron microscopy measurements.

\section{Supporting Information}

TEM image and DLS measurements of gold nanoparticles, stability of gold nanoparticles in citrate buffer, voltammetric responses of a bulk gold electrode, and SEM image of gold nanoparticles attached onto ITO electrodes by successive scans. This material is available free of charge via the Internet at http://pubs.acs.org.

\section{Statement of Competing Interests}

The authors declare no competing financial interests. Commercial equipment or materials are identified in this paper to specify adequately the experimental procedure. Such identification does not imply recommendation or endorsement by the National Institute of Standards and Technology, nor does it imply that the materials or equipment identified are necessarily the best available for the purpose.

\section{List of Abbreviations}

\author{
AuNP-Gold nanoparticles \\ $\mathrm{Au}(\mathrm{III})$-Trivalent gold \\ ITO-IndiumTinOxide \\ DLS-Dynamic Light Scattering \\ TEM-Transmission Electron Microscopy \\ NHE-Normal Hydrogen Electrode \\ CV-Cyclic Voltammetry
}

Ep-Peak Potential.

\section{References}

[1] Shipway, A. N.; Katz, E. and Willner, I., "Nanoparticle Arrays on Surfaces for Electronic, Optical, and Sensor Applications”, ChemPhysChem., 1.18-52.Aug. 2000.

[2] Oyama, M., "Recent Nanoarchitectures in Metal Nanoparticlemodified Electrodes for Electroanalysis”, Analyt. Sci., 26.112.Jan.2010.

[3] Xiao, Y.; Patolsky, F.; Katz, E.; Hainfeld, J. F. and Willner, I., "Plugging into Enzymes: Nanowiring of Redox Enzymes by a Gold Nanoparticle”, Science, 299.1877-1881.Mar.2003.

[4] Liu, S.; Ju, H., "Electrocatalysis via Direct Electrochemistry of Myoglobin Immobilized on Colloidal Gold Nanoparticles”, Electroanal., 15. 1488-1493. Oct.2003.

[5] Goyal, R. N.; Gupta, V. K.; Oyama, M. and Bachheti, N. "Gold Nanoparticles Modified Indium Tin Oxide Electrode for the Simultaneous Determination of Dopamine and Serotonin: Application in Pharmaceutical Formulations and Biological Fluids”, Talanta, 72. 976-983.May.2007.

[6] Daniel, M.-C.; Astruc, D., "Gold Nanoparticles: Assembly, Supermolecular Chemistry, Quantum-Size-Related Properties, and Applications toward Biology, Catalysis, and Nanotechnology”, Chem. Rev. 104. 293-346.Jan.2004.

[7] Finot, M. O.,Braybrook, G. D., and McDermott, M. T., "Characterization of Electrochemically Deposited Gold Nanocrystals on Glassy Carbon Electrodes”, J. Electroanal. Chem., 466, 234-241. Jul.1999.

[8] Dai, X., Compton, R. G., "Direct Electrodeposition of Gold Nanoparticles onto Indium Tin Oxide Film Coated Glass: Application to the Detection of Arsenic (III)”,Analyt. Sci., 22.567570. Apr.2006.

[9] Wang, L., Mao, W., Ni, D., Di, J., Wu, Y., and Tu, Y., “Direct Electrodeposition of Gold Nanoparticles onto Indium/Tin Oxide Film Coated Glass and Its Application for Electrochemical Biosensor”, Electrochem. Commun., 10. 673-676. Apr.2008.

[10] Ivanova, O. S., Zamborini, F. P., "Electrochemical Size Discrimination of Gold Nanoparticles Attached to Glass/IndiumTin-Oxide Electrodes by Oxidation in Bromide-Containing Electrolyte”, Anal. Chem., 82. 5844-5850. Jul.2010.

[11] Masitas, R. A., Zamborini, F. P., “Oxidation of Highly Unstable $<4 \mathrm{~nm}$ Diameter Gold Nanoparticles $850 \mathrm{mV}$ Negative of the Bulk Oxidation Potential”, J. Am. Chem. Soc., 134. 5014-5017. Mar.2012.

[12] Li, Y., Cox, J. T., and Zhang, B.,” Electrochemical Responses and Electrocatalysis at Single Au Nanoparticles”, J. Am. Chem. Soc., 132.3047-3054.Mar.2010.

[13] Bonanni, A., Pumera, M, and Miyahara, Y.,’Influence of Gold Nanoparticle Size (2-50 nm) Upon Its Electrochemical Behavior: an Electrochemical Impedance Spectroscopic and Voltammetric Study”, Phys. Chem. Chem. Phys. 13. 4980-4986. Jan.2011.

[14] Zhou, Y.-G., Rees, N. V., Pillay, J., Tshikhudo, R., Vilakazi, S., and Compton, R. G., "Gold Nanoparticles Show Electroactivity: Counting and Sorting Nanoparticles Upon Impact with Electrodes”, Chem. Commun. 48. 224-226. Jan.2012.

[15] Zhang, J., Kambayashi, M., and Oyama, M. A., "Novel Electrode Surface Fabricated by Directly Attaching Gold Nanospheres and Nanorods onto Indium Tin Oxide Substrate with a Seed Mediated Growth Process”, Electrochem. Commun. 6. 683-688.Jul.2004.

[16] Xiao, X., Bard, A. J., ”Observing Single Nanoparticle Collisions at an Ultramicroelectrode by Electrocatalytic Amplification”, $J$. Am. Chem. Soc., 129.9610-9612. Aug.2007.

[17] Xiao, X., Fan, F.-R. F., Zhou, J., and Bard, A. J., "Current Transients in Single Nanoparticle Collision Events”, J. Am. Chem. Soc., 130.16669-16677. Dec.2008.

[18] Nakagawa, T., Bjorge, N. S., and Murray, R. W.,"

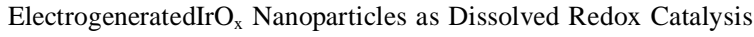
for Water Oxidation”, J. Am. Chem. Soc., 131.15578-15579. Nov.2009.

[19] Kwon, S. J., Fan, F.-R. F., and Bard, A. J., "Observing Iridium Oxide $\left(\mathrm{IrO}_{\mathrm{x}}\right)$ Single Nanoparticle Collisions at Ultramicroelectrodes”, J. Am. Chem. Soc., 132. 13165-13167. Sep. 2010.

[20] Zhou, Y.-G., Rees, N. V., and Compton, R. G., ”The Electrochemical Detection and Characterization of Silver 
Nanoparticles in Aqueous Solution”, Angew. Chem. Int. Ed., 50. 4219-4221. Apr.2011.

[21] Lakbub, J., Pouliwe, A.,Kamasah, A., Yang, C., and Sun, P., "Electrochemical Behaviors of Single Gold Nanoparticles", Electroanal., 23.2270-2274. Oct.2011.

[22] Ward Jones, S. E., Campbell, F. W., Baron, R., Xiao, L., and Compton, R. G., "Particle Size and Surface Coverage Effects in the Stripping Voltammetry of Silver Nanoparticles: Theory and Experiment”, J. Phys. Chem. C,,112. 17820-17827. Nov.2008.

[23] Ivanova, O. S.,Zamborini, F. P., "Size-Dependent Electrochemical Oxidation of Silver Nanoparticles", J. Am. Chem. Soc., 132.7072.Jan.2010.

[24] Busbee, B. D.,Obare, S. O., and Murphy, C. J., ”An Improved Synthesis of High-Aspect-Ratio Gold Nanorods", Adv. Mater., 15. 414-416. Mar.2003.

[25] Kambayashi, M., Zhang, J., and Oyama, M., "Crystal Growth of Gold Nanoparticles on Indium Tin Oxides in the Absence and Presence of 3-Mercaptopropyl-trimethoxysilane”, Cryst. Growth Des.,5. 81-84. Mar.2005.

[26] Cadle, S. H.,Bruckenstein, S.,"Ring-Disk Electrode Study of the Anodic Behavior of Gold in 0.2M Sulfuric Acid",Anal. Chem., 46. 16-20.Jan.1974.
[27] Ogura, K., Haruyama, S., and Nagasaki K., ”The Electrochemical Oxidation and Reduction of Gold", J. Electrochem. Soc., 118. 531-535. Apr.1971.

[28] Shackleford, S. G. D., Baxall, C., Port, S. N., and Taylor, R. J., ”AnIn Situ Electrochemical Quartz Microbalance Study of Polycrystalline Gold Electrodes in Nitric Acid Solution”, J. Electroanal. Chem., 538-539. 109-119. Dec.2002.

[29] Wang, Y.,Laborda, E., Salter, C.,Crossley, A., and Compton, R. G.,"Facile In Situ Characterization of Gold Nanoparticles on Electrode Surfaces by Electrochemical Techniques: Average Size, Number Density and Morphology Determination”,Analyst.,137. 4693-4697.Aug.2012.

[30] Tremiliosi-Filho, G.,Dall'Antonia, L. H., and Jerkiewicz, G., "Growth of Surface Oxides on Gold Electrodes under WellDefined Potential, Time and Temperature Conditions", $J$. Electroanal. Chem., 578. 1-8. Apr.2005.

[31] Conway, B. E., "Electrochemical Oxide Film Formation at Noble Metals as a Surface-Chemical Process", Prog. Surf. Sci., 4. 331452. Aug.1995.

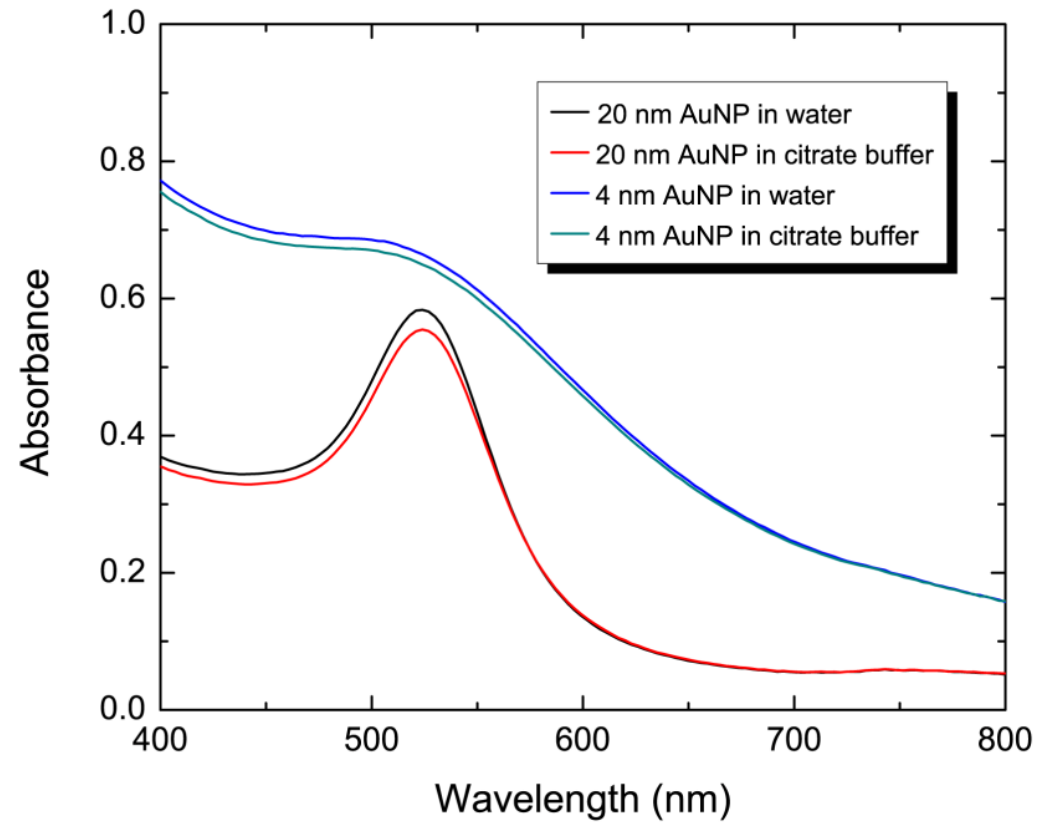

Figure 1S. The stability of gold nanoparticles with nominal diameters $4 \mathrm{~nm}$ and $20 \mathrm{~nm}$ stored in citrate buffer (pH 6.0) for $12 \mathrm{~h}$ was monitored by measuring the surface plasmon resonance bands centered at ca. $517 \mathrm{~nm}$ and $530 \mathrm{~nm}$, respectively

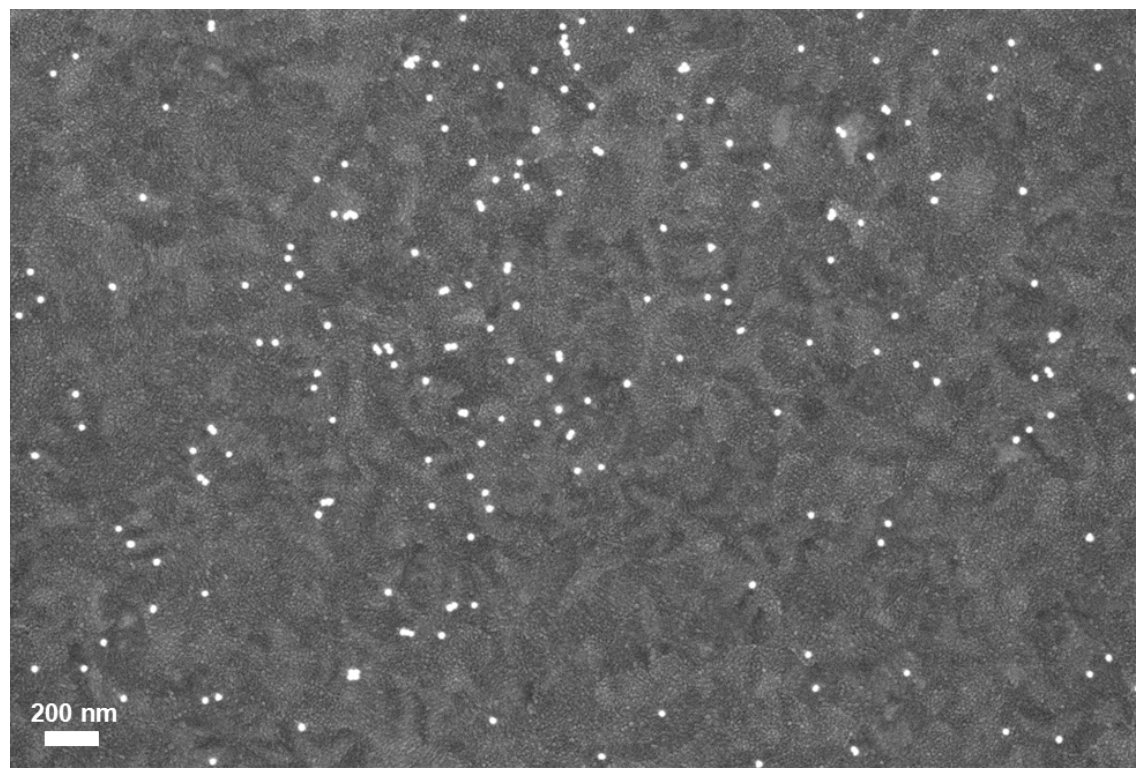

Figure 2S. Scanning electron microscopy image of gold nanoparticles (20 nm nominal diameter) deposited onto an ITO electrode with five successive scans in the potential range from $0 \mathrm{~V}$ to $1.1 \mathrm{~V}$ 


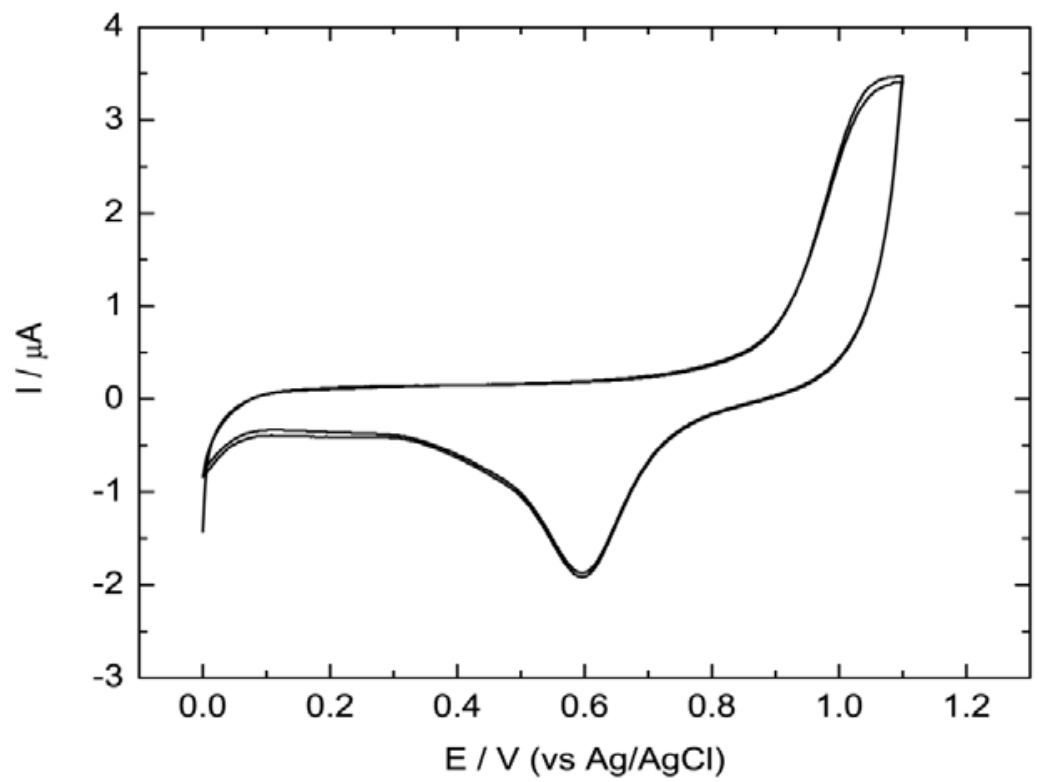

Figure 3S. Cyclic voltammogram of bulk gold electrode (1 mm diameter) in $13.3 \mathrm{mM}$ citrate buffer (pH 6.0) at a scan rate of $60 \mathrm{mV} \mathrm{s}^{-1}$
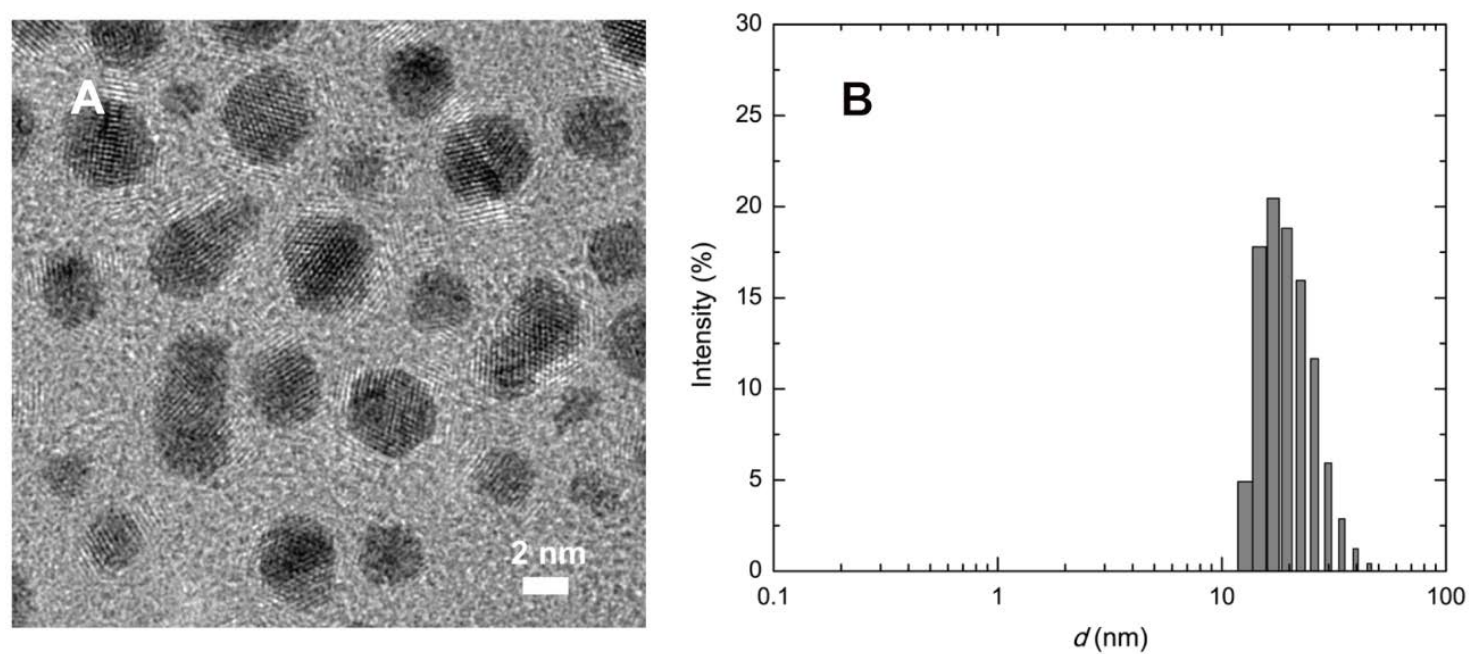

Figure 4S. Transmission electron microscopy image (A) and particle-size distribution from dynamic light scattering measurements (B) for supplied gold nanoparticles used in the experiments 